Йзв. АН Эстонйи. Физ. Матем.. 1989, 38, № 3, 344-347

удК 535.375 .5

Г. ЗАВТ, Имби ТЕХВЕР, В. ХИЖНЯКОВ

\title{
ВОЗБУЖДАЮЩИИ ПРОФИЛЬ РЕЗОНАНСНОГО КОМБИНАЦИОННОГО РАССЕЯНИЯ ТРИГОНАЛЬНОГО КОЛЕБАНИЯ КУБИЧЕСКОГО ЦЕНТРА
}

G. ZAVT, Imbi TEHVER, V. HIZNJAKOV. RESONANTSE KOMBINATSIOONHAJUMISE ERGASTUSPROFIIL TRIGONAALSE VONKUMISE JAOKS KUUBILISE SOMMEETRIAGA LISANDITSENTRIS

G. ZAVT, Imbi TEHVER and V. HIZHNYAKOV. THE EXCITATION PROFILE OF RESONANCE RAMAN SCATTERING ON THE TRIGONAL MODE OF A CUBIC IMPURITY CENTRE

Резонаксное ксмбинационое рассеяние (РКР) широко используется для изучения электронно-колебательного взаимодействия в молекулах и кристаллах. Возбуждающие профили РКР - изменение интенсивностей линий РКР при сканировании частоты падающего света по полосе оптического поглощения - проявляют колебательнуг структуру, обычпо более четкую, чем в соответствующем спектре поглощения. Анализ этой структуры на основе теории возбуждающих профилей РКР $\left[{ }^{1-3}\right]$ позволяет получить детальную информацию о микропараметрах электронно-колебательного взаимодействия $\left[{ }^{2-8}\right]$.

В данной работе проведен модельный анализ возбуждающих профилей РКР неполносимметричных колебаний центров с учетом эффекта Яна-Теллера в вырожденном возбужденном электронном состоянии. Рассматривается поляризованное рассеяние кубических центров в случае возбуждения в резонансе с электронным переходом $A_{1(g) \rightarrow} \rightarrow T_{1(u)}$. Электронно-колебательное взаимодействие в возбужденном электронном состоянии $T_{1}$ описывается в линейном приближении по смещениям ядер матрицей

$$
V=\left(\begin{array}{ccc}
a Q_{0}+b\left(Q_{1}-\frac{1}{\sqrt{3}} Q_{2}\right) & c Q_{5} & c Q_{4} \\
c Q_{5} & a Q_{0}-b\left(Q_{1}+\frac{1}{\sqrt{3}} Q_{2}\right) & c Q_{3} \\
c Q_{4} & c Q_{3} & a Q_{0}+\frac{2 b}{\sqrt{3}} Q_{2}
\end{array}\right),
$$

где $a, b, c$ являются константами взаимодействия с $a_{1}\left(Q_{0}\right)-, e\left(Q_{1}, Q_{2}\right)$ и $\tau_{2}\left(Q_{3}, Q_{4}, Q_{5}\right)$-колебаниями.

Вычисляется возбуждающий профиль РКР первого порлдка на неполносимметричном недиагональном (в смысле вклада в иатрицу $V$ ) $\tau_{2}$-колебании, приводящем к деполяризации рассеяния. О-метим, что возбуждающие профили $a_{1}$ - и $e$-колебаний, как показано в $\left[{ }^{9}\right]$, описываются формулами стандартного метода преобразования [1,2], рассматривавшегося в целом ряде работ (см., напр., $\left.\left[{ }^{10-13}\right]\right)$.

Возбуждающий профиль РКР первого порядка на $\tau_{2}$-колебании частоты $\omega_{k}$ рассчитывается по формуле [ $\left.{ }^{14}\right]$

$$
I_{\perp}(\omega)=\left|A_{\alpha \beta}(\omega)\right|^{2},
$$


гेде

$$
\begin{gathered}
A_{\alpha \beta}(\omega)=c_{k}\left(\bar{n}_{k}+1\right)^{1 / 2} \int_{0}^{\infty} d \tau \exp \left[-i \omega \tau-\gamma \tau+g_{0}(\tau)-\frac{1}{3} g_{1}(\tau)\right] \times \\
\times \int_{0}^{\tau} d s \exp \left[i \overline{\omega_{k}} s+g_{1}(s)+g_{1}(\tau-s)\right]
\end{gathered}
$$

- амплитуда рассматриваемого рассеяния, $\omega=\Omega-\Omega_{0}, \Omega-$ частота возбуждающего света, $\Omega_{0}$ - частота чисто-электронного перехода, $\gamma$ - радиационная ширина возбужденного электронного уровня, $\alpha, \beta=x, y, z$ - декартовы компоненты поляризации падающего $(\alpha)$ и рассеянного $(\beta)$ света $(\alpha \neq \beta)$,

$$
\begin{aligned}
& g_{0}(x)=a^{2}\left(\left\langle Q_{0} Q_{0}(x)\right\rangle-\left\langle Q_{0}^{2}\right\rangle\right), \\
& g_{1}(x)=2 b^{2}\left(\left\langle Q_{1} Q_{1}(x)\right\rangle-\left\langle Q_{1}^{2}\right\rangle\right)
\end{aligned}
$$

- фононные корреляционные функции $a_{1}$ - и $e$-колебаний, равные

$$
g_{m}(x)=\sum_{i} \xi_{m i}\left[\left(\bar{n}_{i}+1\right)\left(e^{i \bar{\omega}_{t} \tau}-1\right)+\bar{n}_{i}\left(e^{-i \bar{\omega}_{t} \tau}-1\right)\right]
$$

$m=0,1, \bar{n}_{i}=\left(\exp \left(-\omega_{i} / k T\right)-1\right)^{-1}, \pm \bar{\omega}_{i}= \pm \omega_{i}-i \Gamma_{i} ; \Gamma_{i}-$ константа затухания нормального колебания $i, \xi_{m i}-$ безразмерные стоксовы потери на данном колебании. Функции $g_{m}(x)$ в каждом конкретном случае могут быть вычислены методами локальной динамики решетки $\left[{ }^{15}\right]$. В данной работе эти функции выбираются из модельных соображений.

Ниже использовались $g_{m}(x)$-функции

$$
g_{m}(x)=\xi_{m}\left(e^{i \bar{\omega}_{m} x}-1\right),
$$

соответствующие модели локальных колебаний в случае нулевой температуры. На рис. 1, 2 представлены модельные расчеты возбуждающих профилей (ВП) $I_{\perp}(\omega)$ РКР первого порядка на $\tau_{2}$-колебании по формуле (2) с учетом (3) и (6).

1. Рис. 1 демонстрирует зависимость ВП РКР $\tau_{2}$-колебания от параметра взаимодействия с $e$-колебанием $\xi_{1}$. Как следует из рис. 1, эта зависимость немонотонная: например, пик при $\omega / \omega_{h}=1$, соответствующий $\tau_{2}$-колебанию, вначале (при $\left.\xi_{1}=0,5\right)$ исчезает, а затем при увеличении . $\xi_{1}$ начинает возрастать.

$\mathrm{Ha}$ рис. 3 также показано немонотонное поведение пиков ВП при увеличении $\xi_{1}$ в случае разных отношений частот $e$ - $\left(\omega_{1}\right)$ и $\tau_{2}$-колебаний $\left(\omega_{k}\right)$. Пиковые интенсивности ВП $\tau_{2}$-колебания на частотах $\omega=n_{0} \omega_{0}+n_{1} \omega_{1}$ и $\omega=\omega_{k}+n_{0} \omega_{0}+n_{1} \omega_{1}$ вычислялись по формулам

$$
\begin{gathered}
I\left(n_{0} \omega_{0}+n_{1} \omega_{1}\right)=\left(\frac{\xi_{0}^{n_{0} \xi_{1}^{n_{1}}}}{n_{0} ! n_{1} !\left(\gamma+n_{0} \Gamma_{0}+n_{1} \Gamma_{1}\right)}\right)^{2}\left|S_{+}\left(n_{1}\right)\right|^{2}, \\
I\left(\omega_{k}+n_{0} \omega_{0}+n_{1} \omega_{1}\right)=\left(\frac{\xi_{0}^{n_{0}} \xi_{1}^{n_{1}}}{n_{0} ! n_{1} !\left(\gamma+\Gamma_{k}+n_{0} \Gamma_{0}+n_{1} \Gamma_{1}\right)}\right)^{2}\left|S_{-}\left(n_{1}\right)\right|^{2},
\end{gathered}
$$

где $n_{0}$ и $n_{1}$ - номера квантов полносимметричной $a_{1}$-моды и неполносимметричной $e$-моды соответственно;

$$
S_{ \pm}\left(n_{1}\right)=\sum_{p=0}^{\infty} \frac{\xi_{1}^{p}}{p !} \sum_{m=0}^{n_{1}}\left(\begin{array}{l}
n_{1} \\
m
\end{array}\right) \frac{\left(-\frac{1}{3}\right)^{m}}{\bar{\omega}_{k} \pm\left(p-n_{1}+m\right) \omega_{1}} .
$$




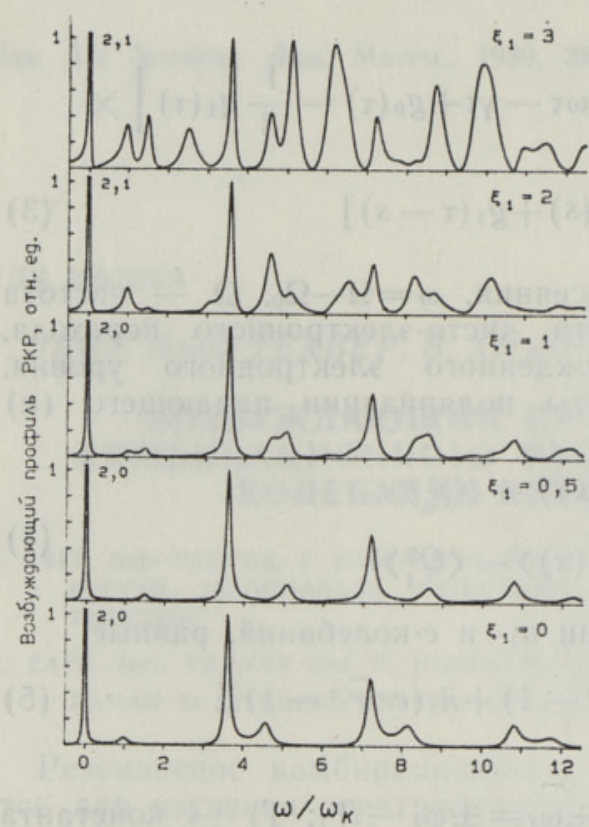

Рис. 1. Возбуждающие профили РКР $\tau_{2}$-колебания при разных стоксовых потерях $\xi_{1}$ на $e$-колебание; $\xi_{0}=2,5 ; \omega_{0}=3,6$; $\Gamma_{0}=0,05 ; \quad \omega_{1}=1,5 ; \quad \Gamma_{1}=0,075 ; \quad \omega_{k}=1 ;$ $\Gamma_{k}=0,1 ; \gamma=0,02$.

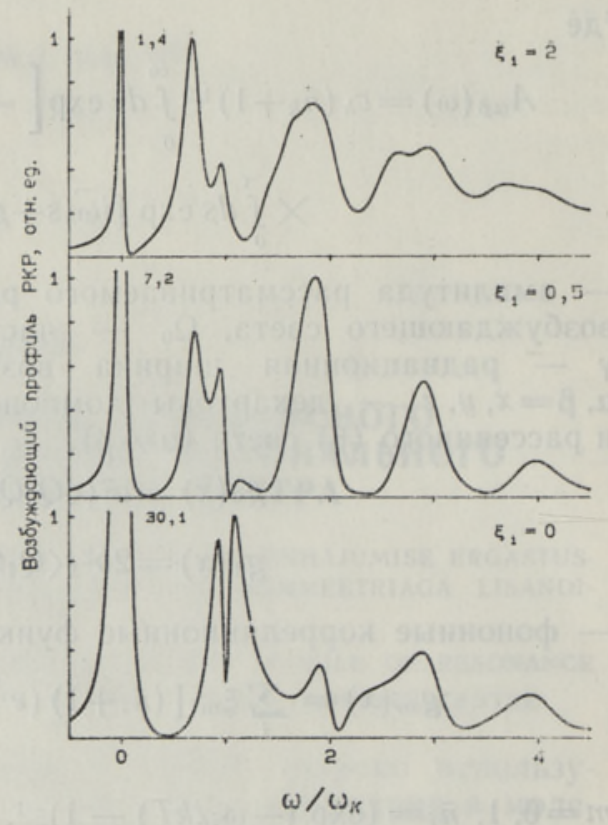

Рис. 2. Возбуждающий профиль РКР $\tau_{2}$-колебания в случае колебательного резонанса $\omega_{k}=\omega_{0}$ (частота $\tau_{2}$-колебания совпадает с частотой полносимметричного $a_{1}$-колебания); $\xi_{0}=2,5 ; \quad \omega_{0}=1 ; \quad \Gamma_{0}=0,1$; $\omega_{1}=0,7 ; \quad \Gamma_{1}=0,075 ; \quad \omega_{k}=1 ; \quad \Gamma_{k}=0,04 ;$ $\gamma=0,02$.
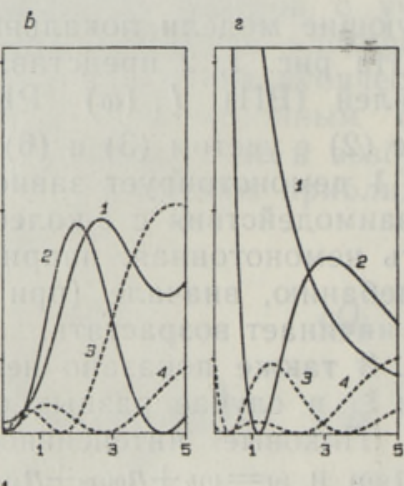

Рис. 3. Отношения пиковых интенсивностей возбуждающего профиля $\tau_{2}$-колебания в зависимо ти от стоксовых потерь на $e$-колебание $\xi_{1}$ в частотах: $a-\omega_{k}, \sigma-\omega_{1}$, $в-\omega_{k}+\omega_{1}, 2-2 \omega_{1}$. Отношения частот $e$-колебания и $\tau_{2}$-колебания $\omega_{1} / \omega_{k}=0,4(I)$, $0,75(2), 1,5 \quad(3), 2,5 \quad(4)$.

Формулы (7)-(9) получены разложением формулы (3) в ряд по степеням $g(x)$ с последующим интегрированием по $\tau$. Представленные на рис. 3 относительные пиковые интенсивности ВП в частотах $\omega=\omega_{k}, \omega_{1}, \omega_{1}+\omega_{k}, 2 \omega_{1}$ равны $R_{ \pm}(m)=\left|S_{ \pm}(m)\right|^{2} /\left|S_{+}(0)\right|^{2}$.

Отметим, что эти зависимости могут быть использованы для определения стоксовых потерь $\xi_{1}$ на $е$-колебания.

2. В частном случае слабого вибронного взаимодействия с неполносимметричными $e$-колебаниями можно приближснно взять $g_{1}(x) \simeq 0$. Тогда амплитуда (3) приводится к виду 


$$
A_{\alpha \beta}(\omega)=c_{k}\left(\bar{n}_{k}+1\right)^{1 / 2} \int_{0}^{\infty} d \tau \exp \left[-i \omega \tau-\gamma \tau+g_{0}(\tau)\right]\left(e^{\overleftarrow{i \omega}_{k} \tau}-1\right),
$$

где первое в скобках слагаемое описывает резонанс по рассеянному, а второе - по возбуждающему свету. (Формула (10) аналогична формуле для амплитуды РКР полносимметричного колебания, т. е. coответствует основной модели.) В этом случае при $\omega_{k}=\omega_{0}\left(\right.$ но $\left.\Gamma_{k} \neq \Gamma_{0}\right)$ возникает интересный эффект расщепления линий ВП первого и второго порядков, обусловленный интерференцией резонансов по возбуждающей и рассеянной частотам (см. рис. 2). Действительно, согласно (10) интенсивность ВП (2) вблизи частоты $\omega \equiv \Omega-\Omega_{0}=0$ равна

$$
I_{\perp} \sim\left|\left(\Delta \omega+i \Delta \Gamma_{k}\right)^{-1}-\xi_{0}\left(\Delta \omega+i \Delta \Gamma_{0}\right)\right|^{2},
$$

где $\Delta \omega=\omega_{0}-\omega, \Delta \Gamma_{l}=\gamma+\Gamma_{l} \quad(l=0, k)$.

При равных ширинах $\Gamma_{0}=\Gamma_{k} \equiv \mathrm{\Gamma}$

$$
I_{\perp} \sim\left(1-\xi_{0}\right)^{2}\left(\Delta \omega^{2}+\Delta \Gamma^{2}\right)^{-1}
$$

- расщепления нет. Однако если $\Gamma_{0} \neq \Gamma_{k}$ и $\Delta \Gamma_{k}=\xi_{0}^{-1} \Delta \Gamma_{0}$, то $I_{\perp}=0$ при $\Delta \omega=0$ и $I_{\perp}>0$ при $\Delta \omega \neq 0$ Следовательно, при $\Gamma_{0} \neq \bar{\Gamma}_{k}$ и $\Delta \Gamma_{k} \simeq \xi_{0}^{-1} \Delta \Gamma_{0}$ линия действительно расщепляется.

При включении взаимодействия с $e$-колебаниями (рис. 2) расщепление исчезает. Поэтому отмеченный интерференционный эффект может наблюдаться только в ВП РКР недиагонального неполносимметричного колебания при совпадении частот последнего с частотой полносимметричного колебания.

\section{Л ИТЕРАТ У РА}

1. Hizhnyakov, V., Tehver, I. // Phys. stat. sol., 1967, 21, 755-768.

2. Tonks, D. L., Page, J. B. // Chem. Phys. Lett., 1979, 66, 449.

3. Siebrand, W., Zgierski, M. Z. // Excited States (ed. E. C. Lim). New York, Academic Press, 1979, 4, 1-136.

4. Максимова Т. Н., Решетняк Н. Б. // ФТТ, 1979, 21, 2677-2684.

5. Ребане Л. А., Хаав А. А. // ФТТ, 1982, 24, 2558-2563.

6. Хаав А. А., Халлер К. Э., Техвер И. Ю., Ребане Л. А. // ФТТ, 1984, 26, 32803289.

7. Максимова Т. И., Минтаиров А. М. // ФТТ, 1987, 29, 1422-1435.

8. Блумберг Г., Ребане Л. // Изв. АН ЭССР. Физ. Матем., 1987, 36, 387-397.

9. Hizhnyakov, V. V., Tehver, I. J. // Proc. Intern. Symp. «Synergetics and Cooperative Phenomena in Solids and Macromolecules». Tallinn, 1982, 133-144.

10. Page, J. B., Tonks, D. L. // J. Chem., Phys., 1981, 75, 5694-5708.

11. Blazej, D. C., Peticolas, W. L. // J. Chem. Phys., 1980, 72, 3134-3142.

12. Chinsky, L., Laigle, A., Peticolas, W. L., Turpin. P.-X. // J. Chem. Phys., 1982, 76, $1-5$.

13. Champion, P. M., Albrecht, A. C. // Ann. Rev. Phys. Chem., 1982, 33, 353-376.

14. Хижняков В., Техвер И. // Изв. АН ЭССР. Физ. Матем., 1989, 38, 25-33.

15. Zavt, G. S., Plekhanov, V. G., Hizhnyakov, V. V., Shepelev, V. V. // J. Phys. C: Solid State Phys., 1984, 17, 2839-2858.

\footnotetext{
Институт физики

Академии наук Эстонской ССР
}

По:тупила в редакцию $23 /$ I 1989 